\title{
TEORÍAS CONTEMPORÁNEAS DEL RECONOCIMIENTO*
}

\author{
CONTEMPORARY THEORIES OF RECOGNITION
}

\section{RICARDO SALAS ASTRAIN**}

\section{RESUMEN}

Este artículo propone, de una manera sucinta, un primer ejercicio crítico de evaluación de las principales tesis que cruzan las teorías contemporáneas del reconocimiento. Relevamos especialmente aquí el libro del filósofo y sociólogo alemán Axel Honneth Lucha del reconocimiento (Kampf um Anerkennung), ligadas a la tradición hegeliana de la 'Annerkenung' y que influye hoy poderosamente en el pensamiento filosófico y en las ciencias sociales. Destacaremos al mismo tiempo dos teorías de tipo hermenéutico presentes en Taylor y Ricoeur, quienes, aceptando en principio el prisma honnethiano, proponen matices diferentes. El propósito específico de este trabajo consiste en presentar algunas de las categorías principales que son parte de las controversias entre estos pensadores. Estas perspectivas teórico-prácticas permiten responder a los conflictos referidos a las minorías étnicas.

Palabras clave: Reconocimiento, filosofía social, justicia, interculturalidad.

\section{ABSTRACT}

This paper proposes, in a succinct manner, a first critical assessment exercise of the main theses crossing contemporary theories of recognition. Here, we specially highlight the book The Struggle for Recognition by the German sociologist and philosopher Axel Honneth, linked to the Hegelian tradition of 'Anerkennung' (Recognition) and

* Este artículo recoge los avances del Proyecto de Investigación financiado por el Fondo de Ciencias y Tecnología, denominado "Teorías contemporáneas del reconocimiento. Una lectura crítica de A. Honneth, Ch. Taylor y P. Ricoeur”. (Fondecyt 1120701).

** NEII-Facultad de Ciencias Sociales de la UCTemuco-Chile. Correo electrónico: rsalas@ uct.cl 
which has a strong influence on philosophical thinking and social sciences. We underline two theories of the hermeneutical type present in Taylor and Ricoeur, who accept, in principle, the honnethian prism and propose different shades. The specific purpose of this paper is to present some of the main categories that are part of disputes between these thinkers. These theoretical and practical perspectives can respond contextually to the conflicts relative to ethnic minorities.

Keywords: Recognition, social philosophy, justice, multiculturalism.

Recibido: 17.10.16. Aceptado: 04.11.16.

\section{INTRODUCCIÓN}

L

A CUESTION DEL reconocimiento se ha vuelto central en el debate de la filosofía política angloamericana y en la filosofía europea continental, y es probable que este interés esté definido por los problemas teóricos que filósofos y cientistas sociales encuentran para dar cuenta de los conflictos que existen al interior de las sociedades contemporáneas, los que no son únicamente de carácter económico y político, sino que refieren a las transformaciones sociales e identitarias de este tiempo. Los esfuerzos filosóficos contemporáneos ligados a la cuestión del reconocimiento no solo son teóricos, sino que son eminentemente prácticos, como indica Ruiz (2013), en cuanto la filosofía política recoge y problematiza críticamente las experiencias socioculturales y políticas ligadas a la injusticia y exclusión de sectores de la sociedad, a las luchas de las minorías étnicas, al feminismo y a la exclusión de los migrantes en el orden social y legal de la sociedad a la que llegan, a la injusticia e inequidad que viven la mayoría de las mujeres y al crecimiento de los nuevos pobres. También posibilita avanzar en una ciudadanía cosmopolita y multicultural, que es parte hoy del contexto actual de una economía y sociedad global que asegura la circulación del dinero y de los objetos, pero impone duras condiciones a hombre y mujeres quienes buscan mejores condiciones de vida en lugares distintos a los que nacieron.

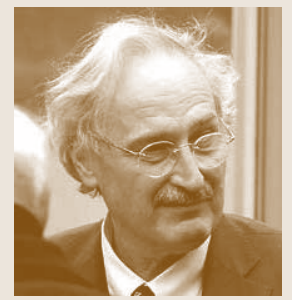

Estas teorías filosóficas y de las ciencias sociales elaboran categorías que resultan pertinentes para explicar estos fenómenos, pero no son compatibles entre sí, y se encuentran dispersas en tradiciones filosóficas y en lenguas diferentes (Salas, 2014). Honneth es conocido por Kampf um Anerkennung. Zur moralischen Grammatik sozialer Konflikte (1992), en la que se releva la noción de "Anerkennung" para fundar una teoría normativa de la sociedad. Él revisita, en diferentes niveles, la tradición hegeliana según el espíritu frankfurtiano, y de cara a los desarrollos actuales de las ciencias sociales críticas. Se sabe que algunas de las principales tesis de esta incipiente teoría 
del reconocimiento están asociadas a la crítica de la obra del sociólogo y filósofo J. Habermas. El análisis crítico de Habermas le permite a Honneth cuestionar la teoría crítica basada en el paradigma de la comunicación e introduce las ciencias sociales críticas en la tercera generación de tal Escuela, destacando la conflictividad propia de las esferas del reconocimiento y asociándolas a las ideas hegelianas expuestas en sus trabajos juveniles de Jena.

La propuesta de Honneth es así el resultado de una reconstrucción crítica que se inicia en los manuscritos del joven Hegel, en el que encuentra tres formas de reconocimiento: el amor (relaciones de cercanía), el derecho (relaciones de igualdad) y la solidaridad o economía (que entrecruza las dos anteriores). No es difícil descubrir allí un esquema inicial que prosigue el pensamiento dialéctico de Marx y de sus seguidores, que buscan, de etapa en etapa, la reconstrucción social de las luchas por el reconocimiento. La tarea de una tal reconstrucción crítica, desde Hegel a Habermas, hace patente a Honneth afirmar el legado cuestionador de las ciencias sociales actuales frente a la sociedad capitalista actual, y propone una crítica social que no sea solo de carácter contextual, sino también que identifique las injusticias con cierto tipo de sociedad, y a la vez dé cuenta de la conciencia de la pluralidad cultural. Se trata asimismo de conectar con la experiencia de la disparidad de los movimientos sociales de emancipación que contribuyen a bajar la expectativa respecto del rol de una crítica social total que derivaba del pensamiento marxista tradicional.

\section{LAS PRINCIPALES TESIS DE LA LUCHA DEL RECONOCIMIENTO}

En este libro referido, Honneth retoma las categorías expuestas en los manuscritos del joven Hegel en Jena, al igual que las referencias elaboradas por G. H. Mead en torno a la identidad y la alteridad en un enfoque de psicología social, para intentar desarrollar "una teoría normativa y sustancial de la sociedad", por la cual se puedan comprender y explicar los conflictos sociales motivados éticamente. En tal noción de lucha se esboza un modelo teórico donde los conflictos sociales ya no están ligados únicamente a intereses económicos o a imposiciones de unas clases o grupos más poderosos, sino que aluden especialmente al modo particular de ser considerados los sujetos al interior de diferentes esferas en una sociedad, en un sentido positivo (reconocimiento) o en un sentido negativo (menosprecio). Se critica tanto una visión especulativa de la filosofía de Hegel como una perspectiva empírica de las ciencias sociales de Mead. Al respecto afirma Honneth: "Ninguno de ellos estaba en condiciones de determinar adecuadamente las expe- 
riencias sociales bajo cuya presión la afirmada lucha por el reconocimiento debe ocasionalmente engendrarse en el proceso histórico; ni en Hegel ni en Mead puede encontrarse una consideración teórica de aquellas formas de menosprecio que, como un equivalente negativo de las correspondientes relaciones de reconocimiento, pueden hacer que los actores sociales experimenten la realidad de un reconocimiento escatimado" (Honneth, [1992] 1997, pp. 115-6). Esta idea es central para pensar los conflictos sociales no como cuestiones exclusivamente políticas, sino que morales, y permite dar cuenta de los daños y sufrimientos sociales que determinan los cursos de acción de los sujetos no reconocidos.

$\mathrm{Al}$ reconstruir un modelo teórico basado en los aportes y limitaciones de ambos pensadores, la categoría de reconocimiento termina siendo reformulada para hacerla apta para la filosofía social y para las ciencias sociales empíricas, en cuanto ambas presuponen determinados valores y normas referidas a la vida intersubjetiva. Sería equívoco, empero, considerar que una teoría de la Anerkennung se basa solo en los manuscritos juveniles de Hegel, sino que ella gira en torno a las posibilidades que tienen las categorías de Mead, y de otros pensadores tales como Marx, Sorel y Sartre, para delinear una nueva teoría crítica, reconstruida sobre la categoría del reconocimiento, lo que permite entender los conflictos sociales a partir de un contexto histórico definido por las luchas por el reconocimiento.

La primera parte de La lucha del reconocimiento, titulada en el original "Historische Vergegenwärtigung: Hegels ursprüngliche Idee", reconstruye los argumentos hegelianos de Jena (especialmente los textos: Sistema de la vida ética y Filosofía del espíritu), en los que, según Honneth, se encuentra un mayor potencial para pensar el carácter normativo de las interacciones sociales. El joven Hegel inspirará y conducirá a Honneth a proseguir la distinción entre tres formas de reconocimiento social mencionadas por Hegel: amor, derecho y solidaridad, que, según los momentos de su dinámica, encierran el potencial de una motivación de los conflictos y de una determinada lucha por lograr el reconocimiento.

El análisis de la realidad social que introduce Hegel permite a Honneth señalar que: "la lucha del reconocimiento no sólo contribuye, como un elemento constitutivo de cualquier proceso de formación, a la reproducción del elemento espiritual de la sociedad civil, sino que actúa también en el sentido de un empuje normativo innovador hacia el desarrollo del derecho en su conformación interna" (Honneth, [1992] 1997, p. 66). Éste es un elemento central, porque el reconocimiento que nace como una categoría jurídica se transforma en la interpretación de Honneth en una conceptualiza- 
ción multidisciplinaria, y que sostiene posibles convivencias de una mirada psicológica, sociológica y económica de la vida social; en otras palabras, permite cruzar el conjunto de la estructura social y evitar los reduccionismos teoréticos de la unidisciplinariedad que abunda en ciencia sociales.

Honneth reelabora y reconstruye el sentido de tales conceptos. Como lo dice una estudiosa de Honneth:

Las respuestas que Hegel halló a estas complejas cuestiones en el curso de la elaboración de sus primeros proyectos de sistema representan el núcleo de su modelo de lucha del reconocimiento, que contiene la arriesgada pero a la vez sugerente idea de que el progreso moral se desarrolla a lo largo de una gradación de tres patrones de reconocimiento de complejidad creciente, entre los cuales se plantea cada vez una lucha intersubjetiva entre los individuos para hacer valer las reivindicaciones de su identidad (Mesquita, en introducción a Honneth, 2009, p. 20).

Es clave en el sentido que refuta ciertas visiones acerca de la sociedad que suponen que para entender los conflictos inherentes de las relaciones sociales hay que separar las relaciones intersubjetivas cotidianas de las relaciones de tipo estructural. Para este enfoque es crucial demostrar que la normatividad social asociada al reconocimiento entre sujetos se va desplegando, de modo que al desvincular las relaciones familiares de las relaciones comunitarias y sociales conlleva la incomprensión de la dinámica social de los sujetos, de la construcción de sus identidades y del modo de asumir los conflictos con los otros.

Volvamos a la hipótesis principal de Honneth, que se deriva estrictamente de la reconstrucción de los esbozos hegelianos del reconocimiento, y que tiene el propósito explícito de elaborar una teoría normativa de los conflictos sociales en la modernidad. Tal perspectiva científica y filosófica ubica su empeño no solo en el terreno filosófico sino en el plano de las ciencias sociales críticas. Como dice Mesquita acerca de los tres escritos de Jena: "Honneth va a encontrar en el Hegel temprano no solo una teoría de la sociedad moderna centrada en el concepto de reconocimiento, sino también una concepción del carácter normativo, basado en expectativas de reconocimiento recíproco, de la lucha social por el reconocimiento" (Mesquita, en Honneth, 2009, p. 22). La hipótesis que ha elaborado Honneth ya no es una tesis únicamente filosófica, por lo tanto no se queda en el mero terreno especulativo y metafísico de Hegel, imposible de ser aceptado hoy día, sino que ella avanza hacia la reconstrucción de una teoría crítica que pueda ser validada por el avance actual de las ciencias sociales críticas. El 
uso que se hace de los resultados de la psicología social y del psicoanálisis permite descubrir las formas de eticidad del lazo encuentro-desencuentro con los otros, y que definen las identidades en permanente construcción.

Su tesis liga la interpretación de la 'Anerkennung' con la obra de Mead, que se condensa sobre todo en la segunda parte del libro ya referido. Este acápite se titula "Systematische Aktualisierung: Die Struktur sozialer Anerkennungsverhältnisse" y analiza en detalle una teoría crítica del reconocimiento de carácter empírico que se encuentra especialmente en la psicología de Mead, y en otros trabajos psicoanalíticos e históricos de la segunda mitad del siglo XX, que van a la zaga de los aportes del psicoanálisis de Winnicott acerca de la constitución del yo en la relación del infante con su madre. Estos nuevos referentes conceptuales y empíricos le permiten delinear los contornos de una teoría crítica que se propone ahora explicar los conflictos de la sociedad en tanto se trata de encuentro entre un yo y los otros, y que le lleva a postular la tesis de que "en la que los procesos de cambio social deben explicarse en referencia a pretensiones normativas, estructuralmente depositadas en la relación de reconocimiento recíproco" (Honneth, [1992] 1997, p. 8). Esta tesis permite una comprensión de los procesos psicológicos que afectan la construcción de la identidad del Yo, y de la apertura al otro y a los otros. Aquí se definen las grandes cuestiones ligadas a la autoestima y a la identidad personal, así como el tema de la intersubjetividad.

La última parte del libro complementa esta tesis poniéndola en relación con algunos otros pensadores de lo social como Marx, Sorel y Sartre. Este acápite, titulado "Sozialphilosophischer Ausblick: Moral und gesellschaftliche Entwicklung", es breve. Permite reelaborar algunos contenidos acerca del reconocimiento y del menosprecio que provienen de los desarrollos de las teorías del conflicto de los tres pensadores mencionados. La crítica de algunas categorías de estos autores permite explícitamente a Honneth comprender los conflictos sociales en relación a las exigencias del reconocimiento, $\mathrm{y}$ a fundamentar un concepto no substancialista de eticidad, que considera fundamental en el desarrollo de las ciencias sociales críticas.

En La lucha del reconocimiento se busca entender el modo en que se constituye la sociabilidad humana y permite comprender de mejor modo la realidad social moderna, destacando el valor del espacio privado y del espacio público, y donde ella aparece cruzada por innumerables formas de reconocimiento y de menosprecios que entregan la clave de por qué los actores sociales se van jugando históricamente por extender los derechos y las nuevas necesidades de reconocimiento; a ello alude el subtítulo del 
libro: "Una gramática moral para los conflictos sociales". Empero Honneth reconoce que la interpretación de los conflictos sociales no va de suyo. Él constata en estos últimos años que existe una variedad de interpretaciones y aplicaciones de dicho concepto (Honneth, 2010, pp. 14-16), donde hay presupuestos que son a veces muy diferentes. De todas las observaciones y comentarios analizados nos parece que dos observaciones teóricas mayores son las que derivan de las perspectivas hermenéuticas de Charles Taylor y Paul Ricoeur: una que proviene del mundo anglosajón y la otra de la tradición francesa.

\section{APORTES HERMENÉUTICOS A LA TEORÍA HONNETHIANA DE LA 'ANERKENNUNG'}

La inexistencia de una teoría unificada del reconocimiento es producto de que subyacen presupuestos filosóficos y científicos disímiles y, tal como lo apunta Ricoeur ([2004] 2006), la cuestión del reconocimiento se enraíza en tradiciones tan diferentes tal como se puede encontrar en Kant y Bergson. Esbozaremos aquí las perspectivas de P. Ricoeur y Ch. Taylor que recogen esta complejidad teorética, ya sea aceptando parte de las tesis honnethianas del reconocimiento, pero también cuestionándola en diversos puntos.

\section{Las tesis de Charles Taylor acerca del reconocimiento}

En los inicios de los años 90, producto de los debates acerca de la teoría rawlsiana de la justicia y ligados a las discusiones políticas acerca de las diferencias, surgieron unos debates en filosofía política y ética en Norteamérica que marcaron el debate posterior del reconocimiento. Charles Taylor inscribe su teoría en un enfoque acerca de la identidad del yo en la modernidad, la que se encuentra en obras tales como: Philosophical Papers I (1985), The Sources of the Self (1986), The Malaise of Modernity (1991). La perspectiva de Taylor se elabora a partir de una tesis acerca de la construcción de la identidad de los sujetos en la modernidad, y de una crítica de las visiones individualistas del hombre, lo que implica sostener que el reconocimiento deriva de una discusión de las miradas comprensivas del bien. Es justamente de esta hermenéutica del análisis del self que se derivarán una serie de consecuencias políticas para comprender el juego entre identidad y diferencia, y que forman parte de su enfoque del reconocimiento.

En este contexto de análisis de la identidad en la modernidad, Ruiz

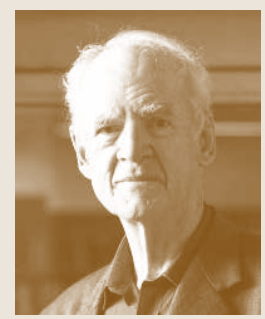

Ch. Taylor

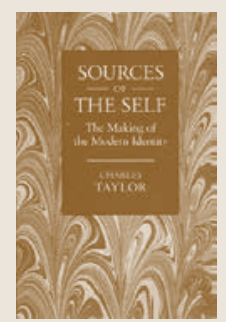

$85 \mid$\begin{tabular}{l|l} 
Atenea 514 \\
\cline { 2 - 2 } & II Sem. 2016
\end{tabular} 
(2013) indica que Taylor se hizo conocido en el debate de los años 90 del siglo pasado, introduciendo una serie de argumentos en los que cuestiona las tesis de un liberalismo neutro (L.1), a favor de otro (L.2) que exige una política del reconocimiento, lo que aparece expresamente en Taylor (1992). En todo caso, sus argumentos filosóficos no tienen que ver en principio con una filosofía social sino con una tesis antropológica central acerca del sentido fuerte de los procesos de valorización en medio de las transformaciones históricas de la modernidad. Por tanto, se trata de definir los alcances de una ética de la autenticidad que le lleva a hacer prevalecer el bien común de una sociedad por encima de los bienes aislados buscados por individuos. En este sentido, no solo hay una crítica al individualismo sino del ideal kantiano de hacer primar la justicia por encima del bien.

El punto de partida de Taylor (1992) asocia reconocimiento con identidad, diciendo que la identidad tiene que ver con el reconocimiento del otro, por los otros. "Me gustaría argumentar -dice- que las identidades en el mundo moderno están cada vez más formadas en esta relación directa con los otros, en un espacio de reconocimiento". De allí se puede decir que la necesidad del reconocimiento es una necesidad humana básica. Él sostiene que el discurso del reconocimiento "se ha vuelto familiar para nosotros en dos niveles: primero en la esfera íntima, donde comprendemos que la formación de la identidad y del yo tiene lugar en un diálogo sostenido y en pugna con los otros significantes. Y luego en la esfera pública, donde la política del reconocimiento igualitario ha llegado a desempeñar un papel cada vez mayor" (p. 59). En la esfera pública se trata de plantear el problema de si en una sociedad democrática puede conciliarse el trato igualitario para todos los individuos con el reconocimiento de las diferencias específicas que dentro de esa misma sociedad se manifiestan.

Taylor analiza, a ese respecto, dos fenómenos ocurridos en estos últimos siglos, cuya comprensión puede contribuir a entender ese problema y aportar a su solución. El primero consiste en el desplome de las jerarquías sociales que servían de base al honor, concepto asociado a la desigualdad, y su reemplazo por el moderno concepto de dignidad, relacionado con la idea de universalidad e igualdad entre los hombres. El segundo fenómeno tiene que ver con la nueva interpretación de la identidad, en el sentido de individualizarla, considerarla como propia de uno mismo, lo que a su vez se asocia a la idea de ser fiel a sí mismo y al particular modo de ser de cada individuo. "Con el tránsito del honor a la dignidad -razona Taylor- sobrevino la política del universalismo que subraya la dignidad igual de todos los ciudadanos, y el contenido de esa política fue la igualación de los derechos 
y los títulos" ([1992] 1993, p. 60). Por contraste, el segundo cambio -el desarrollo del concepto moderno de identidad- hizo surgir la política de la diferencia. Así, la política de la dignidad tiende a establecer un conjunto idéntico de derechos e inmunidades.

En cambio, la política de la diferencia exige que sea reconocida la identidad única de cada individuo o grupo, el ser distinto de los demás. Esta condición de ser distinto es, precisamente, la que según Taylor se ha pasado por alto, ha sido objeto de glosas y asimilada por una identidad dominante o mayoritaria. Por eso mismo, la política de la diferencia ha estado llena, señala, de denuncias de discriminación y de rechazos a la ciudadanía de segunda clase.

En resumidas cuentas, el paradigma del reconocimiento se condensa en la postura de Taylor en tres tópicos: uno, el dolor moral, de identidad; dos, la necesidad igualmente moral del reconocimiento; $y$ tres, la exigencia política, y la correspondiente política del reconocimiento, que es a la vez una política de la identidad y una política de la diferencia. En este plano la postura de Taylor subraya la necesidad de la apertura a las culturas, porque todas las culturas que se han desarrollado merecen nuestro respeto, lo que plantea sendas diferencias con la teoría honnethiana del reconocimiento, ya que involucra la valorización de las identidades y de la diversidad cultural.

\section{El planteo hermenéutico de Paul Ricoeur}

El filósofo Paul Ricoeur desarrolla su tesis particularmente en Parcours de la reconnaissance (2004), donde valoriza el singular aporte de las tesis de Honneth. La cuestión de la teoría del reconocimiento y/o de la política de la diferencia aparece ligada en una tradición hegeliana a la noción de conflicto y de lucha en la terminología de A. Honneth, y con un lugar un tanto insignificante a la experiencia de la reciprocidad, a los denominados "estados de paz y/o de mutualidad", en el vocabulario de P. Ricoeur. Este diálogo es explícito entre ambos, tal como lo indica en la tercera parte de Caminos del reconocimiento: "He concebido esta sección como un diálogo con él; mi contribución va desde la adición de observaciones complementarias hasta la proposición de algunas consideraciones antagonistas, las cuales abrirán, a su vez, el camino a un argumento dirigido contra el énfasis exclusivo puesto en la idea de lucha, a la búsqueda de experiencias de reconocimiento de carácter pacífico" (Ricoeur, [2004] 2006, p. 195). Al lado del reconocimiento en su sentido de lucha, señala Ricoeur, es preciso proponer al don, como una manera de amortiguar el sentido fuerte que se le da a la idea del

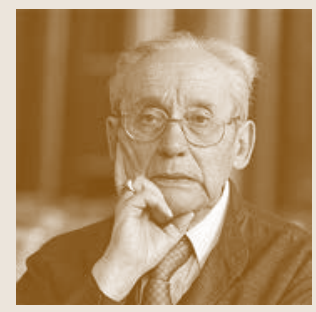

P. Ricoeur

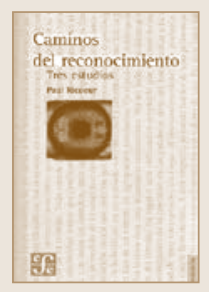

\begin{tabular}{l|l}
87 & Atenea 514 \\
\cline { 2 - 2 } & II Sem. 2016
\end{tabular} 
Paul Ricœur

Parcours de la reconnaissance

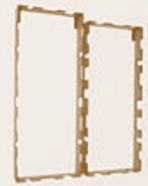

fioessais conflicto. El don implica un estado de paz y su mutualidad se basará en la idea del reconocimiento simbólico. Esta idea implica una dinámica donde el verbo reconocer pasa de la voz activa a la voz pasiva: reconozco algo o a personas, me reconozco a mí mismo y quiero ser reconocido por los otros.

La noción del diálogo y/o debate acerca de la conflictividad y mutualidad del reconocer particularmente es el punto central del disenso entre ambos filósofos. Nos dice Ricoeur en "esta disimetría originaria entre el yo y el otro, disimetría que anula la reciprocidad en cuanto mutualidad" (Ricoeur, [2004] 2006, p. 264), que es parte de una permanente tensión entre la lucha y la paz, como un momento además de la tensión que se da entre el sí mismo y el otro.

Como señala J. Grondin (2013), estos motivos refieren a una gruesa cuestión de una hermenéutica de la historicidad, que a su parecer atraviesa toda la obra ricoeuriana. La hipótesis principal en la obra tardía de Ricoeur permite comprender tanto la unidad del análisis ético-político como además las dificultades existentes en la elaboración de un basamento teórico del reconocimiento en un sentido histórico filosófico. Para lograr esto se exige al menos hacer interactuar tres tradiciones filosóficas europeas, dispares entre sí (Kant, Hegel, Bergson), y cuya disparidad hace difícil, como el mismo Ricoeur lo afirma, elaborar "una teoría unificada del reconocimiento" (2004, p. 28).

El planteamiento de Parcours de la reconnaissance (París, 2004) permite definir el sentido de esta hermenéutica del sí mismo. La idea central es la de pensar la capacidad del hombre de reconocerse responsable de sus actos. Identidad, memoria y promesa son abordados aquí desde la perspectiva del reconocimiento. Finalmente, desde las relaciones con el otro, y más allá de la mutualidad y la disimetría originarias entre el yo y el otro, el filósofo francés nos invita a preservar la justa distancia, garante de la alteridad.

Las ideas generales expuestas de Ricoeur acerca de la Anerkennung confirman en términos generales su cercanía a Honneth, en cuanto a la hipótesis del carácter insuperable de la pluralidad humana en sus transacciones intersubjetivas, de la necesidad de una teoría social de base normativa, y de que las luchas o los conflictos sociales tienen motivos morales, aunque Ricoeur ([2004] 2006), cuestiona fuertemente el excesivo énfasis que hace de la noción de lucha y la poca relevancia de los estados de paz. Un texto explícito de Ricoeur insiste en que el menosprecio no debiera llevarnos a caer en un estilo puramente militante y conflictual del reconocimiento, justamente para conjurar la posibilidad de una "conciencia desgraciada"; nos dice Ricoeur: 
propongo tomar en consideración la experiencia efectiva de lo que llamo estados de paz, y emparejarlos con las motivaciones negativas y positivas de una lucha interminable, como puede ser el análisis en el sentido psicoanalítico del término... la certeza que acompaña a los estados de paz ofrece más bien una confirmación de que no es ilusoria la motivación moral de las luchas del reconocimiento. Por eso, no puede tratarse más que de treguas, de claros, se diría de 'calveros', en los que el sentido de la acción sale de las brumas de la duda con el sello de la acción que conviene ([2004] 2006, pp. 225-6).

En la sección III y IV de Caminos del reconocimiento, Ricoeur destaca otros aspectos relevantes de la Anerkennung y no vacila en aceptarla en lo medular: "En lo esencial, me adhiero a este proyecto. En mi propia terminología, se trata de buscar en el desarrollo de las interacciones conflictuales la fuente de la ampliación paralela de las capacidades individuales evocadas en el segundo estudio bajo el signo del hombre capaz a la conquista de su ipseidad. En el reconocimiento mutuo termina el recorrido del reconocimiento de sí mismo" (Ricoeur, [2004] 2006, p.196). Lo que resulta inviable es quedarse únicamente en la idea de la lucha y que lo lleva a completarla con una reflexión acerca del don: "A partir de la discusión centrada en la idea misma de lucha, derivada de Hegel, intentaremos completar la problemática de la lucha mediante la evocación de las experiencias de paz por las que el reconocimiento puede, si no concluir su recorrido, al menos dejar entrever la derrota de la negación de reconocimiento" ([2004] 2006, p. 197).

El análisis de la argumentación honnethiana es seguida en cada una de las esferas del reconocimiento: de las relaciones cercanas, las relaciones jurídicas y las relaciones solidarias. Son estas últimas las que llaman la atención de Ricoeur, considerando que el análisis de Honneth no es suficientemente amplio en este libro que hemos comentado. Es aquí donde Ricoeur complementa una serie de análisis acerca de la Anerkennung a partir de los órdenes del reconocimiento de J. M. Ferry, de las economías de la grandeza de Bolstanky y Thévenot, y de la política del multiculturalismo de Ch. Taylor. De estas referencias extraídas desde fuera del análisis de Honneth, Ricoeur profundiza la noción de lucha para buscar una alternativa que él considera que se encuentra en las experiencias del reconocimiento mutuo, que descansan en mediaciones sustraídas tanto al orden jurídico como al de los intercambios comerciales.

Las principales ideas de Ricoeur para complementar la teoría de Honneth se sintetizan en la sección $\mathrm{V}$ de Caminos del reconocimiento, y se reducen a lo siguiente: "la experiencia del don, además de su carácter simbólico, 
indirecto, raro, incluso excepcional, es inseparable de su carga de conflictos potenciales vinculada a la tensión creadora entre generosidad y obligación; son estas aporías suscitadas por el análisis ideal-típico del don las que la experiencia del don aporta en su acoplamiento con la lucha por el reconocimiento" (Ricoeur, [2004] 2006, p. 251).

$\mathrm{Y}$, frente a las objeciones de P. Ricoeur acerca de la preeminencia de los estados de guerra por sobre los de paz, Honneth arguye que en Ricoeur predomina aún una mirada fenomenológica, donde se trata de las relaciones entre un sujeto y un alter, mientras que su teoría tiene que ver con la relación que se establece entre sujetos sociales (Marcelo, 2013), de modo que no es solo un asunto de la filosofía hermenéutica, sino de una filosofía de la praxis.

Estas observaciones merecen claramente una serie de aclaraciones, pues justamente la hermenéutica ricoeuriana es una filosofía de la acción entendida como acción sensata.

En Ricoeur encontramos una veta fecunda para reconstruir el espacio teórico de una teoría del reconocimiento, que es a la vez ética y política, y que exige a la vez un trabajo de explicitación de categorías ambiguas y a veces contrapuestas como lo indica Honneth (2010, pp. 35ss), y donde él ha asumido también críticamente las observaciones de P. Ricoeur (Marcelo, 2013). Tal como lo hemos presentado, en la cuestión del reconocimiento se juegan presupuestos que son fenomenológicos y hermenéuticos pero que no siempre son suficientemente explicitados, sobre todo en relación a una ontología social donde la lucha y la paz no se pueden desvincular una de la otra (Renault, 2009).

\section{CONCLUSIONES Y PERSPECTIVAS}

Para terminar indiquemos, a modo de síntesis, que el desafío teorético de entrecruzar los planteamientos ricoeurianos y taylorianos respecto del reconocimiento es que las posturas de ambos filósofos convergen en una hermenéutica del sí, que contribuyen a re-elaborar narrativamente la experiencia de sentido de los sujetos en una sociedad moderna; los dos aportan unos aspectos teóricos acerca de la reconstrucción de la identidad del sujeto en un relación intersubjetiva en medio de los contextos diferenciados de un mundo moderno en cambio, y consideran que la cuestión del reconocimiento es relevante. En ambos los supuestos hermenéuticos implícitos suponen una fenomenología del "entre", no solo un entre antropológico del 
yo y del otro, sino un entre que permite establecer una reciprocidad entre comunidades en búsqueda de sus identidades; en este sentido, en ambos el enfoque permite elaborar una teoría crítica de las interacciones culturales, propia de un pensar intercultural (Salas, 2014).

El principal aporte de Taylor y Ricoeur reside en elaborar una propuesta contraria al subjetivismo e individualismo que considera que el sentido de la experiencia moral no es más que la opción de cada cual, y un correctivo a todas las concepciones liberales basadas principalmente en el resguardo de los derechos de los individuos, y por ello se elabora una argumentada crítica al universalismo pregonado por las teorías procedimentales que provienen de la herencia kantiana y se plasma en el proyecto de un Diskursethik. Para ambos autores, la experiencia del sentido refiere a un concepto de la moral, donde ella no se puede desligar de la eticidad, y en este énfasis reside parte del diferendo con Honneth. Por ello se trata de una ética de la autenticidad en el caso de Taylor, y de una "pequeña ética del sî" en el caso de Ricoeur, que pueda responder a las dinámicas de las subjetividades que hacen frente a las exigencias presentes en la sociedad contemporánea.

La deuda que tiene la filosofía contemporánea del reconocimiento con estos dos autores, a diferencia de Honneth, radica en que este último explicita una sistemática teoría del reconocimiento que no requiere categorías específicas que permitan pensar la diversidad cultural, ni las resistencias que tienen las culturas frente al avance de una modernidad basada en la racionalidad instrumental, y el papel que les cabe a las comunidades. Por ella esta hermenéutica del sujeto, del sí mismo y del self, es claramente convergente con una propuesta intercultural basada en la permanente reflexividad de todo sujeto. En este sentido, la corrección hermenéutica -que no rechaza la propuesta de Honneth-insiste que una teoría crítica del reconocimiento necesita completarse a partir de un análisis más fino de las culturas, de sus historicidades y narrativas específicas.

La teoría del reconocimiento de Honneth expresada en Kampf um Anerkennung marca así un punto de inflexión de la ética y la política. Éste es un pensamiento dialéctico, como lo indicamos, que sigue, de etapa en etapa, la lucha por el reconocimiento y lo dirige ante todo desde la humillación, es decir, de las respectivas formas negativas del reconocimiento que son maltrato/violación; desposesión de los derechos/exclusión e indignidad/ injuria. Es una gramática moral de los conflictos sociales porque la teoría sirve para aprender a interpretar, es decir calificar y así justificar los conflictos sociales, deletreándolos como justos: los no-reconocidos, menospreciados y humillados aprenden a darse cuenta gracias a ella de lo injusto de 
su situación para indignarse y levantarse de la opresión que significa su no-reconocimiento.

Estas ideas resuenan profundamente en los mundos latinoamericanos de vida, porque justamente en ellos las prácticas sociales, políticas y religiosas apuntan normalmente a definir el estatuto de la vida social, donde no se considera de un modo central el valor de la identidades de todos los sujetos, y proseguimos viviendo en sociedades con profundas desigualdades y discriminaciones (Sauerwald y Salas, 2016). Señalar estos sufrimientos sociales y culturales posibilita y legitima la posibilidad de la rebelión y de la resistencia para avanzar en el proyecto de construir una sociedad justa.

No queremos terminar sin aludir a los sufrimientos sociales que caracterizan las historias locales y nacionales. Si pensamos hoy en la situación que se vive en la macrorregión sur de Chile y que caracteriza a los mapuches en su Wallmapu (país mapuche), queda en evidencia una falta generalizada de reflexión teórico-práctica que apunte al reconocimiento recíproco. En tal sentido, falta aún mucho por avanzar en países donde se ha instalado una larga tradición de no-discusión acerca del reconocimiento de las relaciones que establecen los sujetos y las colectividades. La política de la identidad y de la diversidad se vuelve problemática en esos territorios por una larga historia de asimetrías y de negación que cruza completamente el campo económico, político y cultural.

Este diálogo replanteado en el terreno político exige una otra modalidad, más paciente, para entender a los otros no sólo desde las propias articulaciones discursivas, sino desde las prácticas en pos de afirmar las relaciones intersubjetivas dinámicas y las identidades en reconstrucción. En este sentido, Honneth y los dos hermeneutas comentados aquí tienen razón. Pensar el reconocimiento requiere mediaciones ético-políticas. Un diálogo ético-político intercultural colabora en el difícil arte de comprender los propios procesos discursivos y práxicos que exigen a los otros, pero el diálogo en política se da siempre de un modo mucho más opaco, porque implica asumir los intereses espúreos y las expectativas y ambiciones de sujetos y colectividades.

\section{REFERENCIAS}

Becka, M. (2016). "Reconocimiento como base de la interculturalidad - hacia una práctica transformadora”. En Sauerwald, G. y Salas, R. (Eds.), La cuestión del reconocimiento en América Latina (pp. 65-83). Münster: LIT Verlag. 
Fornet-Betancourt, R. (2012). Interculturalidad, crítica y liberación. Aachen, Alemania: Wissenschaftsverlag Mainz.

Gracia J. (2012). "Identidades complejas y dinámicas. Redescubriendo el potencial hermenéutico de la filosofía política de Ch. Taylor”, Revista Española de Ciencia Política, 28, 11-30.

Gómez-Müller, A. (2009). La reconnaissance: réponse a quels problemes? Paris, Francia: L'Harmattan.

Grondin, J. (2013). Paul Ricoeur. París, Francia: P.U.F.

Honneth, A. (1992). Kampf um Anerkennung-Zur moralischen Grammatik sozialer Konflikte. Frankfurt am Main: Suhrkamp (trad. español Barcelona, 1997).

- (2009). Crítica del agravio moral. Buenos Aires, Argentina: FCE.

. (2010). Reconocimiento y menosprecio. Sobre la fundamentación normativa de una teoría social. Madrid: Katz Editores.

Marcelo, G. (2013). "Ricoeur and the Utopia of Mutual Recognition”. Études Ricouriennes / Ricour Studies, 2, 110-133.

Mesquita, M. (2009). “Introducción”. En Honneth, A., Crítica del agravio moral. Buenos Aires, Argentina: FCE.

Renault, E. (2008). Souffrances sociales. Philosophie, psychologie et politique. Paris, Francia: La Découverte.

. (2009). "Reconnaissance, lutte, domination : le modèle hégélien”. Politiques et Sociétés, 28(3), 23-43.

Ruiz, C. (2013). "Modernidad e identidad en Ch. Taylor". Revista de Filosofía, 69, 227-243.

Ricoeur, P. (2004). Parcours de la reconnaissance. Paris, Francia: Stock (trad. español, Madrid, 2006).

Salas, R. (2014). "Debates teórico-metodológicos acerca de reconocimiento e interculturalidad”. Revista Faro, 20, 55-65.

Sauerwald, G. (2008). Reconocimiento y liberación. Axel Honneth y el pensamiento latinoamericano. Berlin, Alemania: Lit Verlag.

Sauerwald, G. y Salas, R. (Eds.) (2016). La cuestión del reconocimiento en América Latina. Münster: LIT Verlag.

Taylor, Ch. (1992). Multiculturalism and the Politics of Recognition. Princeton, USA: Princeton University Press. (trad. español, 1993). 